\title{
Critical Challenges for Primary Students
}

Tami McDiarmid, Rita Manzo, and Trish Musselle. British Columbia: The Critical Thinking Cooperative, 1999, 66 pp. ISBN O-86491-147-5

Critical challenges engage primary students in prioritizing ideas, proposals and suggestions. They enable students to go beyond mere listing of positive and negative choices. When making decisions, students review good and poor choices alongside possible and interesting ones. They also consider all avenues and then systematically review their choices. This approach to critical thinking, challenges primary students to improve their problem-solving skills since systematic and reflective considerations often override initial and emotional responses.

Critical Challenges for Primary Students is a new addition to the Critical Challenges Across the Curriculum series which provide a cluster of teacher resources aimed at infusing critical thinking in all areas of the curriculum. The actual teaching of intellectual skills and the embedded curriculum approach, are hallmarks of this particular book making it a good choice to add to teacher resource selections on critical thinking, in primary grades.

The critical challenges for primary students described in the book contain a number of tasks that are based on the premise that, critical thinking is a way of teaching the content of the curriculum. The tools of critical thinking are systematically defined and taught within students' explorations. The challenges call for teachers to nurture competent critical thinkers right in the classroom. Each critical challenge listed in the text follows a prescriptive format. The challenges begin with critical questions. Overviews are then provided describing the lessons and featured student activities.

The critical challenges also include suggested activity sections following general lesson layouts. These activity sections embody the challenges and the teaching of relevant components. Icons on the right side panel, indicate specific critical thinking tools as they are utilized by the group. An evaluation section follows each lesson and lists assessment criteria for the teacher. Extensions and references follow each critical challenge and provide further consideration and application of the lesson's key features.

The noted extensions are clear and concise. They suggest relevant activities and invite students to engage in the assessment of the effectiveness of their completed activities. Evaluation criteria are specific and include ways to match chosen criteria with subsequent formal evaluation. Reference materials included for each challenge are also brief, specific and useful for students and teachers. The Critical Challenge activities are easy to explore because of the format in which they are presented. Human nature, moral dilemmas, family issues, and teaching and learning from others, are content areas considered in the challenges. These topics appeal to primary students who are encouraged to blend background knowledge with group choices through the prescribed critical challenges. 
The challenges encourage students to de-emphasize emotional reactions to new problems while the exercises help them learn how to adopt an open-minded attitude to problem-solving. Students are required to continue looking for alternatives even if they do not appear at first glance. A rigorous review of alternatives is necessary when the obvious choice is not a satisfactory one. Discussions, charts, webs, and roleplay situations guide children through possible choices.

The activities contained in the challenges also ask children to rank information. Students review brainstorm and web activities to develop priorities and group their action plans. The students are responsible for avoiding the merely obvious priorities and choosing the most valid priorities for completing the critical challenge activities. This increases opportunities for cognitive development by the students, who are expected to choose priorities by asking questions of their group members. The queries lead to prioritization and subsequent action plans. Alternate plans, the role of consequences, and requisite time lines are reviewed by the student work groups when choosing the final solution.

Exploring the realm of possibility is another critical thinking component that is developed throughout the lessons. The ultimate outcome and its related string of possible smaller outcomes, are critically reviewed by group members. Clarifying the group's thinking about the chosen outcome helps members to move systematically to the outcome's completion. When applicable, unclear individual and group outcomes are readily identified by students, as the challenges are completed and evaluated. Students' problem-solving skills are extended when students are required to ponder whether their choices are reversible or not. Immediate choices and long-term plans are considered for positive and negative features. Overall group dynamics are also reviewed and extended through the critical thinking activities. Students use consensus to determine and utilize rules and time lines. Decisions are upheld if they benefit the greatest number of students involved. The group's understanding of the solution is pivotal for the actualization of outcomes.

Primary children are, by nature, explorers and will respond positively to the format of the critical challenges described in the book. The cognitive abilities of students will likely expand through deliberate focus on the components of critical thinking as laid out in this intriguing activity guide for primary grades.

Laurie Bizero

University of Windsor 